\title{
Neonatal bilateral lidocaine administration into the ventral hippocampus caused postpubertal behavioral changes: An animal model of neurodevelopmental psychopathological disorders
}

\author{
Vanessa Blas-Valdivia \\ Edgar Cano-Europa \\ Adelaida Hernández-García \\ Rocio Ortiz-Butrón \\ Departamento de Fisiología "Mauricio \\ Russek Berman", Escuela Nacional \\ de Ciencias Biológicas, I.P.N., Carpio \\ y Plan de Ayala, México
}

\begin{abstract}
Our aim was to investigate if neonatal bilateral administration of lidocaine into the ventral hippocampus would cause behavioral changes related to schizophrenia. A neonatal ventral-hippocampal lesion (nVH lesion) was made with lidocaine in Wistar male pups. Two groups were formed, the first received lidocaine $(4 \mu \mathrm{g} / 0.3 \mu \mathrm{L})$ and the second an equal volume of vehicle. At day 35 and 56, both groups were tested for social contact, immobility caused by clamping the neck and dorsal immobility, locomotor activity in an open field, and tail flick (TF) latency after a painful heat stimulus. All animals were then killed. Coronal cuts $(7 \mu \mathrm{m})$ of the brain were obtained and each brain section was stained with cresyl violet-eosin. The animals which received the $\mathrm{nVH}$ lesion with lidocaine had decreased social interaction at both ages. The rats with lesions, only at day 58 postnatal, increased their distance traveled and ambulatory time, with a decrease in their nonambulatory and reset time. The rats with lesions had a longer duration of immobility caused by clamping the neck and a longer dorsal immobility at both days 34 and 57 compared to control rats. The lidocaine-treated group spent less time to deflect the tail compared to the control group at postpubertal age. The neonatal bilateral administration of lidocaine into the ventral hippocampus caused some alterations, such as chromatin condensation, nucleolus loss, and cell shrinkage, but glial proliferation was not seen. Neonatal bilateral lidocaine administration into the ventral hippocampus caused postpubertal behavioral changes.
\end{abstract}

Keywords: lidocaine, hippocampus, neonatal lesion, behavior, animal model, psychopathological disorders

\section{Introduction}

Lidocaine is a local anesthetic widely used in the clinic because of the blockade of sodium influx in nociceptive fibers. High lidocaine doses have been shown to be toxic because it can cause the cauda equina syndrome and transient neurological symptoms after spinal anesthesia. ${ }^{1}$ It also produces sedation, tremulousness, and dysphoria in humans. ${ }^{2}$ Studies of the central nervous system of the rat show that lidocaine activates limbic structures such as the hippocampus and amygdale ${ }^{3}$ and causes neuronal damage in those structures. ${ }^{4}$

Both the amygdala and hippocampus are implicated in psychiatric disorders as autism, ${ }^{5}$ attention-deficit hyperactivity, ${ }^{6}$ and schizophrenia. ${ }^{7-9}$

Many epidemiological and clinical correlation studies show that obstetric complications, in utero exposure to ethanol, severe malnutrition, and viral infection are risk factors for the development of schizophrenia. ${ }^{10,11}$ It has been hypothesized that schizophrenia is 
a disease related to abnormal neurodevelopment of cortical and subcortical circuitry in which the amygdala and hippocampus participate..$^{10,12}$

Rats with an ibotenic acid-caused neonatal excitotoxiclesion in the ventral hippocampus show, in adulthood, a variety of behavioral abnormalities, such as a deficit in the prepulse inhibition of startle, ${ }^{13}$ latent inhibition, ${ }^{14}$ impaired social behavior, ${ }^{15}$ working memory deficit, ${ }^{16}$ hyperlocomotion in an open-field test, ${ }^{17}$ high immobility response caused by clamping the neck, ${ }^{18}$ and hyperalgesia. ${ }^{19}$ All these behaviors show parallels to schizophrenia. However, the neonatal excitotoxic-lesion with ibotenic acid causes gliosis, a process absent in schizophrenic patients. ${ }^{8}$ The aim of our work was to investigate if the neonatal bilateral administration of lidocaine into the ventral hippocampus caused behavioral changes related to schizophrenia.

\section{Material and methods}

\section{Animals and housing}

All animal procedures were done in accordance with the guidelines of the Laws and Codes of Mexico in The Seventh Title of the Regulations of the General Law of Health Regarding Health Research.

The neonatal ventral-hippocampal lesions (nVH lesion) with lidocaine were made in Wistar male pups on day 7 when the pups weighed 15-17 g. The neonatal administration was made as described previously. ${ }^{20}$ Briefly, pups were anaesthetized by hypothermia and immobilized in a stereotaxic frame. To enable stable fixation of the head a silicone mould was constructed into which rat pups of a specific head size fit. A midline skin incision was made, the skull was perforated using a $1.0 \mathrm{~mm}$ dental drill, and lidocaine $(4 \mu \mathrm{g} / 0.3 \mu \mathrm{L}$ over $2 \mathrm{~min})(n=8)$ or vehicle $(0.1 \mathrm{M}$ phosphate-buffered saline, $\mathrm{pH} 7.4)(n=8)$ was injected bilaterally through $0.33 \mathrm{~mm}$ stainless steel cannula into the ventral hippocampus (coordinates: AP $-3.0 \mathrm{~mm}, \mathrm{ML} \pm 3.5 \mathrm{~mm}, \mathrm{DV}-5.0 \mathrm{~mm}$ relative to Bregma). Subsequently, the skin was stitched and the animals were returned to their nurse.

\section{Behavioral procedure}

All behavioral testing was done between 9 am and 12 pm and was recorded on videotape using a VHS video camera (NV-N3000PN, Panasonic).

Because it is known that signs and symptoms of schizophrenia emerge after puberty, we analyzed the behavioral test at both prepuberal (P33-P35) and postpuberal (P56-58) ages.

On days 33 and 56, rats were brought, in individual plastic cages, to the testing area, which was in the same building and floor as the colony room. Two animals from the same experimental group were placed in the testing area and ten minutes of their social interaction was videotaped and automatically analyzed by the Videomex V apparatus (Columbus Instruments, Columbus, $\mathrm{OH}$ ). After each trial, the rats were returned to the colony room and the area was cleaned with a detergent. The test time of day was balanced across experimental groups.

On days 34 and 57, the animals were tested for immobility caused by clamping the neck and dorsal immobility.

On days 35 and 58, rats were brought, in individual plastic cages, to the testing area. An animal was placed on the open field for six minutes and locomotor behavior was recorded every three minutes. After this, tail flick (TF) latency after a painful heat stimulus was recorded. There were three minutes between each test on each animal.

When the behavioral test was completed, the rat was returned to the colony room and the work area was cleaned with a neutral detergent. The test time of the day was balanced across the experimental groups.

\section{Behavioral testing}

\section{Social contact test (P33 and P56)}

The general design was adapted from Rex. ${ }^{21}$ Twenty-four hours before the testing, animals were singly housed in a metallic cage $(20 \times 30 \times 18 \mathrm{~cm})$ with food and water $\mathrm{ad}$ libitum. The cages were located together in racks to maintain auditory and olfactory contact. The test was made in an open arena $(60 \times 60 \times 30 \mathrm{~cm})$. The floor was made of smooth black wood. The light conditions were comparable to the light intensity in the housing room; two 39-W overhead fluorescent bulbs were suspended $180 \mathrm{~cm}$ above the center of the field and provided $210 \mathrm{Lx}$ at the floor. The social behavior was measured using a video image analyzer (Videomex-V) over ten minutes. The social contact program provides a simple method for recording the interaction between two animals. It gives the total number of contacts during the experiment and the total time the animals have been in contact.

\section{Immobility induced by clamping}

the neck (P34 and P57)

The immobility response was made by attaching a clamp (a 5-cm alligator clip with its tips covered with masking tape to avoid any injury to the rat's skin) between the base of the skull and the back of ears with the pressure being sufficient to lift the whole animal by the clamp. A second clamp was attached to the ventral part of the neck to increase the pressure in this area. The animal was then inverted into 
a supine position. The duration of immobility was measured from the time the animal was placed on its back until it recovered the prone position or until 180 s had elapsed. ${ }^{18,22}$

\section{Dorsal immobility (P34 and P57)}

The animal was gently grasped by the skin at the back of the neck and lifted off its feet $30-40 \mathrm{~cm}$ over the experimental table, avoiding disturbing the animal's breathing and using only enough pressure to lift the whole rat body. When animal stopped moving, the time of immobility was recorded until the animal made escape-like movements directed at the experimenter's hand or until 180s had elapsed. ${ }^{22}$

\section{Open-field test in an unknown environment (P35 and P58)}

This test has been regularly used to assess emotion in rodents. ${ }^{23}$ The animal was transferred to the testing room and immediately placed on the middle of the open field (blackpainted wooden box, $60 \times 60 \times 30 \mathrm{~cm}$ ). The light conditions were comparable to the social contact test. The spontaneous locomotor activity in an unfamiliar environment was measured using a video image analyzer in two sessions of three min each. This keeps track of the distance the animal travels, the amount of time spent traveling, the amount of time spent in a nonambulatory activity, and the amount of time resting. It also displays the path tracings during the session.

\section{Nociceptive test (P35 and P58)}

The tail flick (TF) test was made using restraining tubes constructed from Plexiglas pipe $(6.3 \times 20 \mathrm{~cm})$ with circular Plexiglas lids attached to the ends. Small holes were drilled in the front and sides of the tubes to provide adequate ventilation. TF latencies were measured using a tail flick apparatus (Columbus Instruments, Columbus, $\mathrm{OH}$ ) that delivers radiant heat. The light source positioned below the tail was focused on the proximal third of the rat's tail. Deflection of the tail activated a photocell and automatically terminated the trial. The TF latency represents the period of time from the beginning of the trial to the tail deflection. A cut-off time of $30 \mathrm{~s}$ was used to prevent tissue damage.

\section{Brain histology}

All animals were anesthetized with sodium pentobarbital (35 mg/kg ip) 48-h after the testing day and they were transcardially perfused, first with $0.9 \%$ saline and then with $10 \%$ formaldehyde. The brains were removed and were embedded in paraffin. Coronal cuts of $7 \mu \mathrm{m}$ were obtained from -1.8 to $-4.8 \mathrm{~mm}$ from Bregma. ${ }^{24}$ Each brain section was stained with cresyl violet-eosin, dehydrated, and mounted with resin.

\section{Statistical analysis}

All results are presented as mean \pm SE. Statistical analysis for all behavioral tests was evaluated by two-way ANOVA, Student's $t$-test, and a post hoc Newman-Keuls. The two factors evaluated in ANOVA were age and treatment. $P<0.05$ was considered statistically significant.

\section{Results}

\section{Social contact test}

Figure 1 shows that animals that received the $n V H$ lesion with lidocaine had decreased social interaction at two ages (panel A, between age $F_{1,12}=1.20, P=0.30$; between treatment $F_{1,12}=59.05, P<0.0001$; and interaction age $\times$ treatment $\left.F_{1,12}=0.04, P=0.85\right)$ without affecting the number of contacts (panel B, between age $F_{1,12}=1.43, P=0.25$; between treatment $F_{1,12}=0.03, P=0.87$; and interaction age $\times$ treatment $F_{1,12}=0.01, P=0.91$ ).
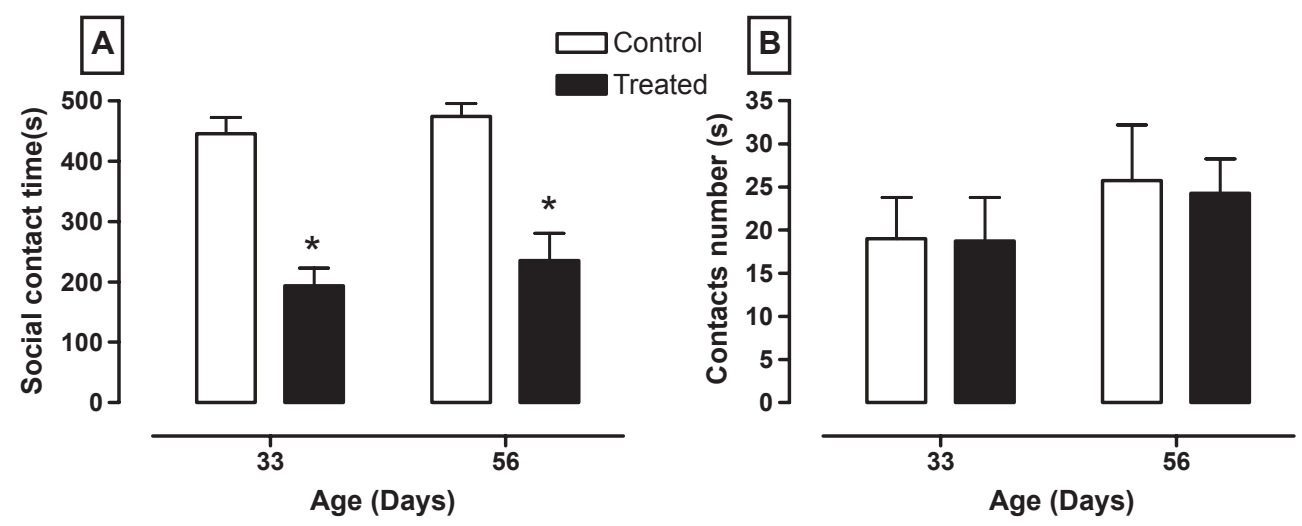

Figure I Effect of neonatal bilateral administration of lidocaine into the ventral hippocampus on social contact time (A) and contact number (B) in I0 minutes of behavioral test. Note: $* P<0.05$ vs control group at the same age. 


\section{Immobility responses}

Immobility responses were modified after the $\mathrm{nVH}$ lesion with lidocaine. Thus, panel A of Figure 2 shows that rats with lesions had a longer duration of immobility caused by clamping the neck at both 34 and 57 days compared to control rats (between age $F_{1,28}=8.31, P<0.009$; between treatment $F_{1,28}=18.36, P<0.001$; and interaction age $\times$ treatment $F_{1,28}=1.55, P=0.23$ ). Also, panel B of figure 2 shows that treated rats had higher dorsal immobility in both ages (between age $F_{1,28}=8.44, P<0.01$; between treatment $F_{1,28}=23.16, P<0.001$; and interaction age $\times$ treatment $\left.F_{1,28}=1.884, P>0.05\right)$.

\section{Open-field test}

Figure 3 shows how the $\mathrm{nVH}$ lesion with lidocaine affects the variables of the open-field test such as the distance traveled (between age $F_{1,28}=15.98, P<0.001$; between treatment $F_{1,28}=5.85, P<0.025$; and interaction age $\times$ treatment $F_{1,28}=2.45, P=0.13$ ), reset time (between age $F_{1,28}=10.19$, $P<0.005$; between treatment $F_{1,28}=11.36, P<0.005$; and interaction age $\times$ treatment $F_{1,28}=2.20, P=0.15$ ), ambulatory time (between age $F_{1,28}=13.29, P<0.009$; between treatment $F_{1,28}=11.31, P<0.005$; and interaction age $\times$ treatment $F_{1,28}=0.06, P=0.80$ ), and nonambulatory time (between age $F_{1,28}=1.69, P=0.20$; between treatment $F_{1,28}=5.60, P<0.05$; and interaction age $\times$ treatment $\left.F_{1,28}=4.15, P=0.055\right)$. It shows that all animals reduced their distance traveled at postpubertal age (panel A), whereas rats with lesions increased their locomotor activity and distance traveled at day 58 compared with its respective control. In contrast, reset time is reduced in animals with lesions at postpubertal age and increased in control animals at the same age (panel B). Animals with lesions spent more time exploring and had more ambulatory time at both ages, whereas control rats spent less ambulatory time at day 58 (panel C). Panel D shows that control animals had less nonambulatory time at postpubertal age, whereas rats with lesions had this variable enhanced at the same ages.

\section{Tail flick}

Figure 4 shows how the $\mathrm{nVH}$ lesion lidocaine lesion affects tail flick latency (between age $F_{1,28}=21.69, P<0.001$; between treatment $F_{1,28}=5.51, P<0.05$; and interaction age $\times$ treatment $F_{1,28}=4.19, P<0.05$ ). We saw that age increases tail flick latency in all animals, however the lidocaine-treated group spent less time to deflect the tail compared to the control group at postpubertal age.

\section{Brain histology}

The brain histology is shown in Figure 5. It shows that the $\mathrm{nVH}$ lesion with lidocaine yields the dorsal and ventral portion of hippocampus morphologically intact without gliosis (panel A). Amplification of the CA3 hippocampal region (panels B and C) shows that control animals have normal neurons with a distinguishable nucleus, nucleolus, and a high ratio nucleus/cytoplasm (panel B), whereas neonatal bilateral administration of lidocaine into the ventral hippocampus caused a reduction of neurons with some alterations such as chromatin condensation, nucleolus loss, and cell shrinkage, but glial proliferation was not seen (panel C).

\section{Discussion}

Lidocaine is the most popular of local anesthetics and is widely used in the clinic. However, high doses of this drug
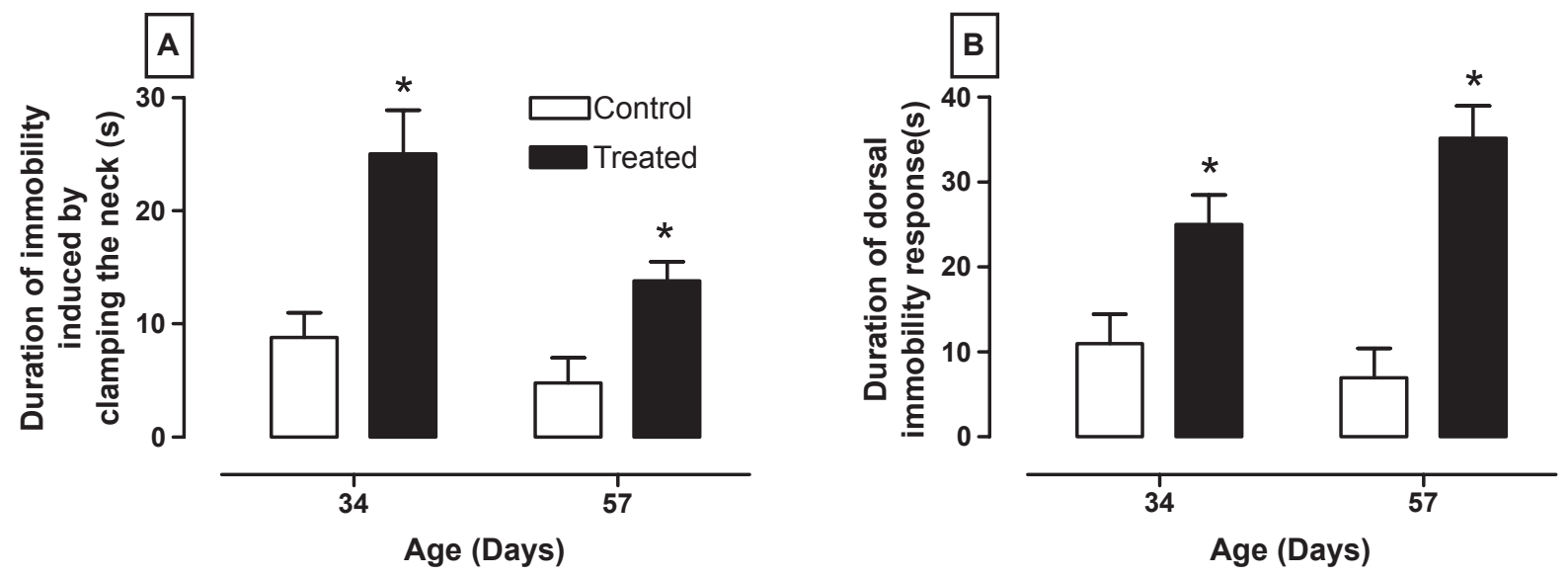

Figure 2 Effect of neonatal bilateral administration of lidocaine into the ventral hippocampus on durations of immobility caused by clamping the neck (A) and dorsal immobility (B).

Note: ${ }^{*} P<0.05$ vs control group at the same age. 

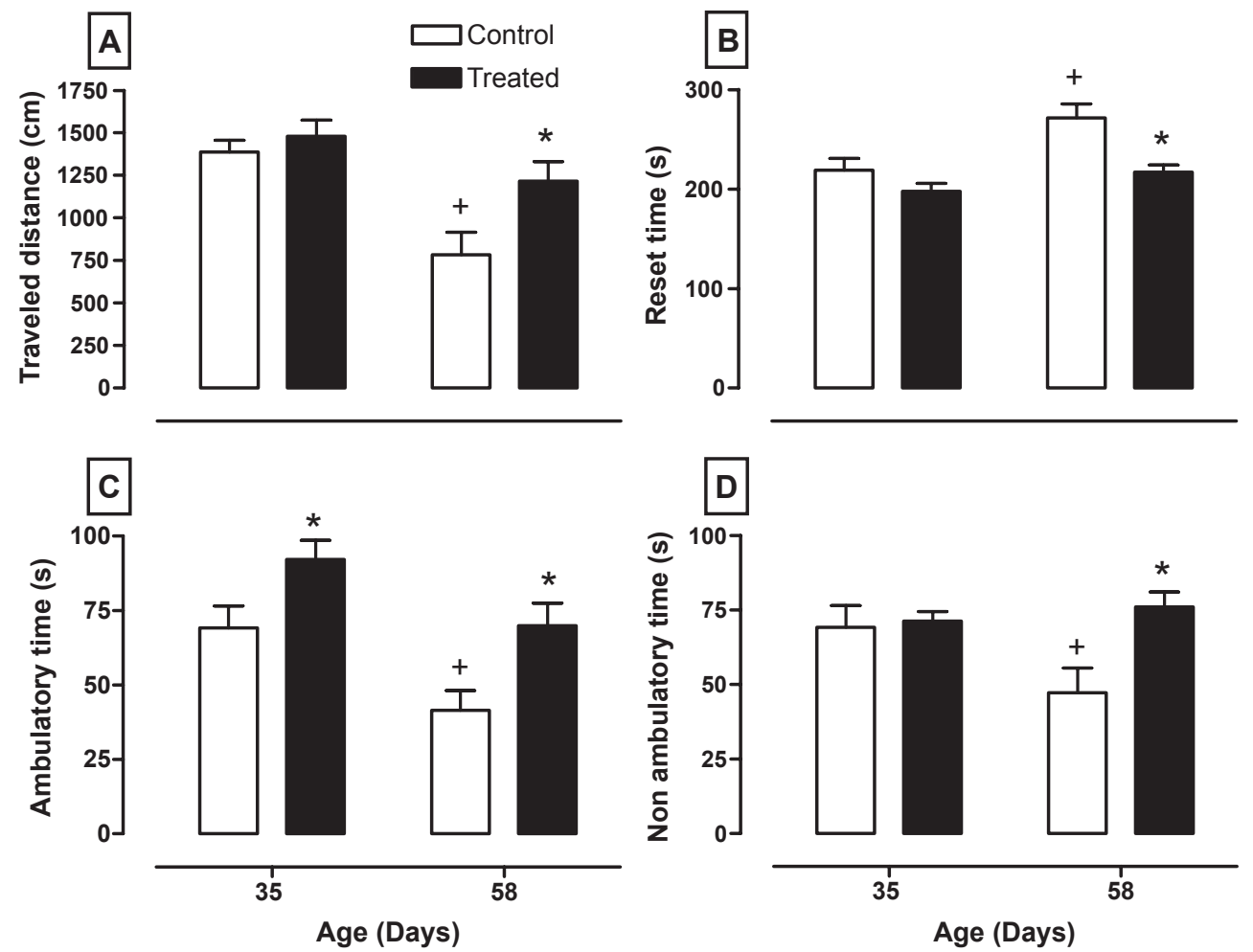

Figure 3 Effect of neonatal bilateral administration of lidocaine into the ventral hippocampus on traveled distance (A), amount of time resting (B), as well as amount of ambulatory (C) and nonambulatory (D) time during six minutes of the open-field test in an unknown environment.

Notes: $* P<0.05$ vs control group at the same age; ${ }^{+} P<0.05$ vs same group vs age of 35 days old.

administrated systemically in adult rats cause adverse effects as seizures, asphyxia, tachycardia, and death. ${ }^{2}$ Post and coworkers studying lidocaine-caused kindling demonstrated that chronic lidocaine administration causes activation of the amygdala and hippocampus, ${ }^{3}$ which causes seizure and behavioral changes related to schizophrenic symptoms. ${ }^{25}$ Additionally, our research group demonstrated that one injection of lidocaine caused selective neuronal damage in the hippocampus and amygdale. ${ }^{4}$ Moreover, there are many studies focusing on neonatal damage of restricted limbic structures in rats, thus neonatal excitotoxic lesions of the rat ventral hippocampus lead, in early adulthood, to altered behaviors that are closer to symptoms present in psychopathological diseases. Our objective of this research is to determine if the $\mathrm{nVH}$ lesion with lidocaine caused behavioral changes in Wistar rats by testing social interaction, open-field activity, dorsal immobility responses and that caused by clamping the neck, and the tail-flick test.

The social interaction test is a simple protocol that has been used in rats to test for anxiety in a novel environment. In this work, we found that the lidocaine-treated group decreased social interactions at both ages. This finding indicates that the $\mathrm{nVH}$ lesion with lidocaine altered neuronal pathways related to the hippocampus because this structure participates in sequences of social behavior (starting, stopping, or continuous social contact). ${ }^{26}$ Our results are in concordance with others, though they used an $\mathrm{nVH}$ lesion caused by ibotenic acid in Sprague-Dowley rats, ${ }^{15}$ however did not find any postpubertal difference in the social interaction test using Wistar rats that had an ibotenic acid-caused neonatal lesion in the $\mathrm{VH} .{ }^{27}$ Reduction in social interaction can be related to hypersensitivity to dopamine ${ }^{28}$ because the social behavior of animals is sensitive to dopaminergic and

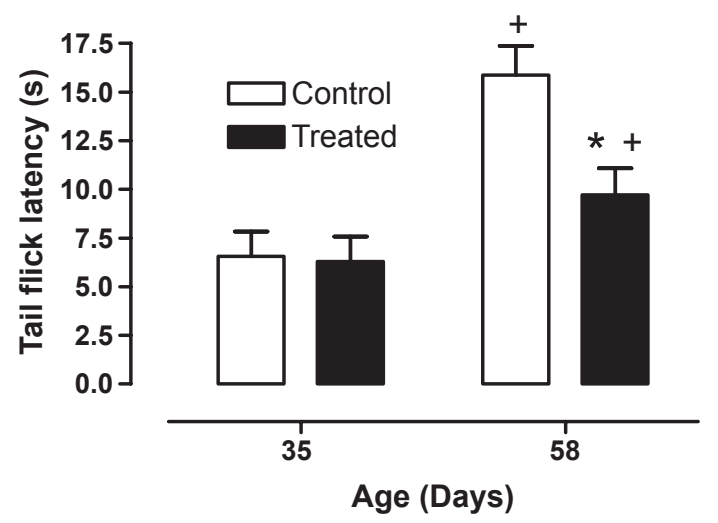

Figure 4 Effect of neonatal bilateral administration of lidocaine into the ventral hippocampus on tail flick latency during nociceptive test.

Notes: $* P<0.05$ vs control group at the same age; ${ }^{+} P<0.05$ vs same group vs age of 35 days old. 

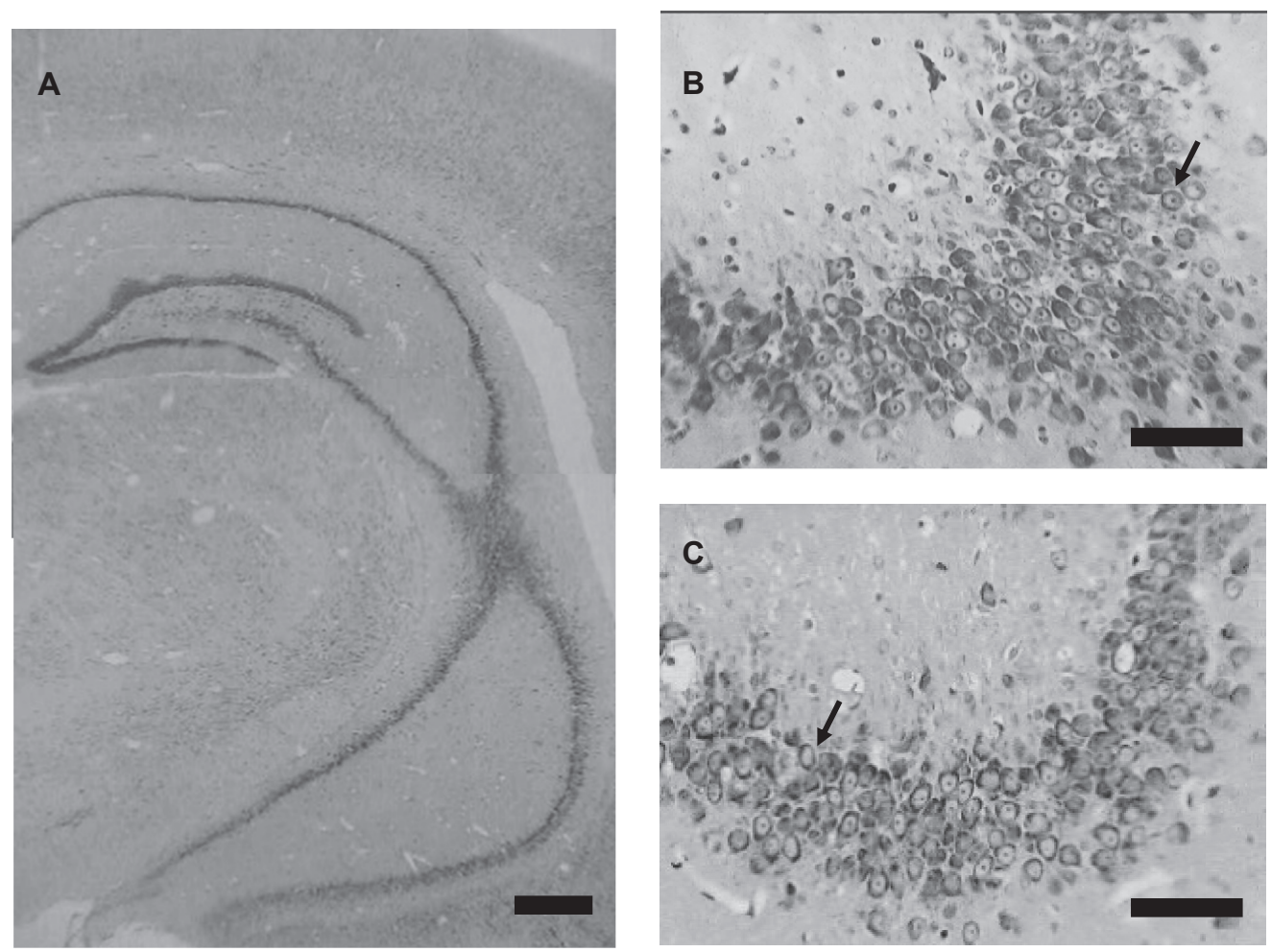

Figure 5 Photomicrographs of coronal section through the brains of animals which had neonatal bilateral administration of lidocaine into the ventral hippocampus (A and C) and control animals (B). Panel A shows that lidocaine-treated animals had the dorsal and ventral portion of hippocampus morphologically intact without gliosis. When we amplified the CA3 hippocampal region (B and C) we observed that control animals (B) had normal morphology represented by neurons with distinguishable nucleus, nucleolus, and a high ratio of nucleus/cytoplasm (arrows in B demonstrated normal neuron of this region), whereas neonatal bilateral administration of lidocaine into the ventral hippocampus caused a mild reduction of neurons with some alterations such as chromatin condensation, nucleolus loss, and cell shrinkage, but no observed glial proliferation (arrows in $\mathrm{C}$ demonstrated alterations).

Note: Bar in A represents $250 \mu \mathrm{m}$ and bars in B and C represent $50 \mu \mathrm{m}$.

glutamatergic manipulation caused by D1 and D2 agonists, and phencyclidine. ${ }^{29}$

In nature, the immobility response is part of an antipredatory behavior and is used by prey to reduce the probably of predator attacks. Experimentally, immobility responses can be produced in the laboratory where they can be caused by fear or by using a dopamine antagonist (such as haloperidol). The immobility caused by clamping the neck and dorsal immobility were enhanced in animals with an $\mathrm{nVH}$ lesion with lidocaine evaluated at the two ages. Though Flores and coworkers made lesions in Sprague-Dawley rats, our result are in accordance with theirs. ${ }^{18}$ It is possible that activation of a dopaminergic mesolimbic pathway, which arrives at the accumbens nucleus from the ventral tegmental area (VTA), modifies the duration of immobility responses because the animals with higher locomotor response to a novel environment increase the basal firing and bursting activity of dopaminergic neurons in the VTA. ${ }^{30}$ In addition, immobility responses are keen behavioral tests that show a general idea of the status of the dopaminergic system since the prepuberal age, because immobility responses are modulated by structures such as the basal nuclei and nucleus accumbens. $^{22,31}$

The open-field test in an unknown environment is another test to evaluate emotion in rats. We found that the $\mathrm{nVH}$ lesion with lidocaine causes an increase of the distance traveled because reset time decreased and both ambulatory and nonambulatory times are enhanced. Again, these results are in accordance with those obtained in the heuristic model of schizophrenia, ${ }^{10,17}$ but not in accordance with results obtained using Wistar rats with an $\mathrm{nVH}$ lesion with ibotenic acid. ${ }^{32}$ The $\mathrm{nVH}$ lesion affects dopaminergic afferences to the prefrontal cortex, which increases locomotor activity in a novel environment at P58 and is related to a higher anxiety level. ${ }^{33}$ Moreover, the $\mathrm{nVH}$ lesion causes a corticolimbic-pathway hyperactivity that increases locomotor activity caused by dopamine release in the nucleus accumbens. ${ }^{34}$

Another test in our model was the latency tail flick. We found that lidocaine only modifies this response at day 56 . These results are in accord with results obtained in SpragueDowley rats with neonatal lesions made with ibotenic acid. ${ }^{19}$ Dopamine is involved in a nociception mechanism ${ }^{35}$ and 
our model and heuristic models of schizophrenia modify the dopaminergic system related to nociception. There are several studies of the functional role of basal nuclei in the somatosensorial information process about thermal pain stimulus. ${ }^{36}$ There is evidence that a change in pain experience occurs in conjunction with certain psychiatric disorders. However, the results found in the neonatal administration of lidocaine to the ventral hippocampus in Wistar rats and in the heuristic model of schizophrenia has demonstrated several alteration findings opposite to "schizophrenic analgesia". ${ }^{37}$

The morphological study reveals that neonatal lidocaine administration in the ventral hippocampus causes a reduction of normal morphological neurons in the CA3 hippocampal region. These results suggest that the lidocaine dose used in this study causes damage. We previously proposed that lidocaine causes cytotoxicity by NMDA-receptor activation ${ }^{38}$ in limbic structures as the hippocampus and amygdala. ${ }^{4}$ The lidocaine dose used by us is higher than when used as a sodium-channel blocker. ${ }^{39,40}$ Moreover, the $\mathrm{nVH}$ lesion made with lidocaine did not cause gliosis as shown in postmortem studies of schizophrenic patients. ${ }^{8}$

All behavioral tests in this study and the morphological evidences support the idea that an $\mathrm{nVH}$ lesion caused by lidocaine in Wistar rats could be a novel model for a neurodevelopmental hypothesis to study schizophrenia.

\section{Acknowledgments}

This study was partially supported by CONACyT grants 53227 and SIP-IPN 20070318 and 2008038. R.O-B is fellow of EDI, DEDICT-COFFA-I.P.N. and SNI, A.H-G is fellow of EDI and DEDICT-COFFA-I.P.N., E.C-E is fellow of CONACyT, V.B-V is fellow of PIFI-COFFA-I.P.N and CONACyT. We thank Dr. Ellis Glazier for editing this English language text.

\section{References}

1. De Jong RH. Local Anesthetics. St Louis, MO: Mosby; 1994. p. 250-303.

2. Steen PA, Michenfelder JD. Neurotoxicity of anesthetics. Anesthesiology. 1979;50:437-453.

3. Post RM, Kennedy C, Shinohara M, et al. Metabolic and behavioral consequences of lidocaine-kindled seizures. Brain Res. 1984;324:295-303.

4. Blas-Valdivia V, Cano-Europa E, Hernandez-Garcia A, Ortiz-Butron R. Hippocampus and amygdala neurotoxicity produced by systemic lidocaine in adult rats. Life Sci. 2007;81:691-694.

5. Courchesne E. Brainstem, cerebellar and limbic neuroanatomical abnormalities in autism. Curr Opin Neurobiol. 1997;7:269-278.

6. Marsden CA. Dopamine: the rewarding years. $\mathrm{Br} J$ Pharmacol. 2006;147(Suppl 1):S136-S144.

7. Bogerts B, Lieberman JA, Ashtari M, et al. Hippocampus-amygdala volumes and psychopathology in chronic schizophrenia. Biol Psychiatry. 1993;33:236-246.
8. Harrison PJ. The neuropathology of schizophrenia: A critical review of the data and their interpretation. Brain. 1999;122:593-624.

9. Schmajuk NA. Hippocampal dysfunction in schizophrenia. Hippocampus. 2001;11:599-613.

10. Lipska BK, Weinberger DR. To model a psychiatric disorder in animals schizophrenia such a reality test. Neuropsychopharmacology. 2000;23:223-239

11. Stefan M, Travis M, Murray RM. An Atlas of Schizophrenia. London: Informa HealthCare; 2002.

12. Carlsson A. The neurochemical circuitry of schizophrenia. Pharmacopsychiatry. 2006;39(Suppl 1):S10-S14.

13. Lipska BK, Swerdlow NR, Geyer MA, Jaskiw GE, Braff DL, Weinberger DR. Neonatal excitotoxic hippocampal damage in rats causes post-pubertal changes in prepulse inhibition of startle and its disruption by apomorphine. Psychopharmacology (Berl). 1995;122:35-43.

14. Grecksch G, Bernstein HG, Becker A, Hollt V, Bogerts B. Disruption of latent inhibition in rats with postnatal hippocampal lesions. Neuropsychopharmacology. 1999;20:525-532.

15. Becker A, Grecksch G, Bernstein HG, Hollt V, Bogerts B. Social behaviour in rats lesioned with ibotenic acid in the hippocampus: quantitative and qualitative analysis. Psychopharmacology (Berl). 1999;144:333-338.

16. Lipska BK, Aultman JM, Verma A, Weinberger DR, Moghaddam B. Neonatal damage of the ventral hippocampus impairs working memory in the rat. Neuropsychopharmacology. 2002;27:47-54.

17. Sams-Dodd F, Lipska BK, Weinberger DR. Neonatal lesions of the rat ventral hippocampus result in hyperlocomotion and deficits in social behaviour in adulthood. Psychopharmacology (Berl). 1997;132:303-310.

18. Flores G, Silva-Gomez AB, Barbeau D, Srivastava LK, Zamudio S, De La Cruz Lopez F. Effect of excitotoxic lesions of the neonatal ventral hippocampus on the immobility response in rats. Life Sci. 2005;76:2339-2348.

19. Al Amin HA, Atweh SF, Jabbur SJ, Saade NE. Effects of ventral hippocampal lesion on thermal and mechanical nociception in neonates and adult rats. Eur J Neurosci. 2004;20:3027-3034.

20. Lipska BK, Jaskiw GE, Weinberger DR. Postpubertal emergence of hyperresponsiveness to stress and to amphetamine after neonatal excitotoxic hippocampal damage: a potential animal model of schizophrenia. Neuropsychopharmacology. 1993;9:67-75.

21. Rex A, Voigt JP, Gustedt C, Beckett S, Fink H. Anxiolytic-like profile in Wistar, but not Sprague-Dawley rats in the social interaction test. Psychopharmacology (Berl). 2004;177:23-34.

22. Zamudio S, Fregoso T, Miranda A, De La Cruz F, Flores G. Strain differences of dopamine receptor levels and dopamine related behaviors in rats. Brain Res Bull. 2005;65:339-347.

23. Walsh RN, Cummins RA. The open-field test: a critical review. Psychol Bull. 1976;83:482-504.

24. Paxinos G, Watson C. The rat brain in stereotaxic coordinates. New York: Academic Press; 1999.

25. Post MR, Kopanda RT, Lee A. Progressive behavioral changes during chronic lidocaine administration: relationship to kindling. Life Sci. 1975; 17:943-950.

26. Maaswinkel H, Gispen WH, Spruijt BM. Executive function of the hippocampus in social behavior in the rat. Behav Neurosci. 1997;111:777-784.

27. Daenen EW, Wolterink G, Gerrits MA, Van Ree JM. The effects of neonatal lesions in the amygdala or ventral hippocampus on social behaviour later in life. Behav Brain Res. 2002;136:571-582.

28. Wan RQ, Corbett R. Enhancement of postsynaptic sensitivity to dopaminergic agonists induced by neonatal hippocampal lesions. Neuropsychopharmacology. 1997;16:259-268.

29. Sams-Dodd F. Phencyclidine-induced stereotyped behaviour and social isolation in rats: a possible animal model of schizophrenia. Behav Pharmacol. 1996;7:3-23.

30. Marinelli M, White FJ. Enhanced vulnerability to cocaine selfadministration is associated with elevated impulse activity of midbrain dopamine neurons. J Neurosci. 2000;20:8876-8885. 
31. Meyer ME, Cottrell GA, Van Hartesveldt C. Intracerebral haloperidol potentiates the dorsal immobility response in the rat. Pharmacol Biochem Behav. 1993;44:157-160.

32. Daenen EW, Wolterink G, Gerrits MA, Van Ree JM. Amygdala or ventral hippocampal lesions at two early stages of life differentially affect open field behaviour later in life; an animal model of neurodevelopmental psychopathological disorders. Behav Brain Res. 2002;131:67-78.

33. Carter CJ, Pycock CJ. Behavioural and biochemical effects of dopamine and noradrenaline depletion within the medial prefrontal cortex of the rat. Brain Res. 1980;192:163-176.

34. Del Arco A, Mora F. Prefrontal cortex-nucleus accumbens interaction: In vivo modulation by dopamine and glutamate in the prefrontal cortex. Pharmacol Biochem Behav. 2008;90:226-235.

35. Hagelberg N, Jaaskelainen SK, Martikainen IK, et al. Striatal dopamine D2 receptors in modulation of pain in humans: a review. Eur $J$ Pharmacol. 2004;500:187-192.
36. Chudler EH. Response properties of neurons in the caudate-putamen and globus pallidus to noxious and non-noxious thermal stimulation in anesthetized rats. Brain Res. 1998;812:283-288.

37. Lautenbacher S, Krieg JC. Pain perception in psychiatric disorders: a review of the literature. J Psychiatr Res. 1994;28:109-122.

38. López-Galindo GE, Cano-Europa E, Ortiz-Butron R. Ketamine prevents lidocaine-caused neurotoxicity in the CA3 hippocampal and basolateral amygdala regions of the brain of adult rats. $J$ Anesthesia. 2008; In Press.

39. Raley-Susman KM, Kass IS, Cottrell JE, Newman RB, Chambers G, Wang J. Sodium influx blockade and hypoxic damage to CA1 pyramidal neurons in rat hippocampal slices. J Neurophysiol. 2001;86:2715-2726.

40. Waraczynski M. Lidocaine inactivation demonstrates a stronger role for central versus medial extended amygdala in medial forebrain bundle self-stimulation. Brain Res. 2003;962:180-198. 